\title{
Examining the Role of Social Media Analytics in Providing Competitive Intelligence:
}

\section{The Impacts and Limitations}

\author{
Jiwat Ram, La Rochelle Business School, Excelia, France \\ Changyu Zhang, IBBS Suzhou, Jiangsu, China
}

\begin{abstract}
This study examines the role of social media analytics (SMA) in providing competitive intelligence (CI). Building on CI theory, the data from qualitative semi-structured interviews with respondents belonging to social media, manufacturing, telecommunication, IT, and service industries were analyzed using Nvivo coding and matrix queries. The results show that SMA provides an expanded CI beyond the previous limits of customers/markets and competitors, including insights on supply chains, costs, and information-flow. Moreover, SMA-driven CI can provide visibility to supply chain uncertainties enabling improvements in demand planning and inventory management. SMA can provide CI about competitors' strengths and weaknesses and customers' dynamics; however, the bi-directional nature of CI could be determinantal if SM-linked customers are not educated/kept informed. Matrix query results illuminate the differences/similarities in respondents' views. Academically, the study shows that SMA provides expanded CI to businesses beyond previously known scope of competitor analysis.
\end{abstract}

\section{KEYWORDS}

Business Intelligence, Competitive Intelligence, Social Media

\section{INTRODUCTION}

The use of social media (SM) in business and everyday life is increasing exponentially. A recent report shows that there are 3.196 billion SM users globally (representing an annual growth of 13\%), with $79 \%$ of the global internet population (estimated at 4.021 billion in 2018) and $90 \%$ of brands using SM to build brand awareness (Chaffey, 2018). These staggering figures point to the potential value of SM for businesses. In particular, given the pervasiveness of SM use among multiple tiers of society, SM data can provide valuable business intelligence about customers, including their demographics and psychographics, purchasing habits, preferences, and behavioral intentions (He et al., 2017). User-generated data from SM, including users' geolocations, opinions, and preferences, can reveal valuable information about customers' tastes, thoughts, and behaviors, constituting an important source of analytics to obtain competitive intelligence (CI) and other types of business intelligence for decision-makers (Shollo \& Galliers, 2016).

Pellissier and Nenzhelele (2013) define CI as: 
a process or practice that produces and disseminates actionable intelligence by planning, ethically and legally collecting, processing and analysing information from and about the internal and external or competitive environment in order to help decision-makers in decision-making and to provide a competitive advantage to the enterprise. ( $p .7)$

Given the increased competition and associated technological developments (e.g., big data analytics) that enable the collection and processing of large volumes of multicontextual data, CI has assumed center stage, arguably becoming a core business strategy to enhance market position and profitability (Lee, 2018). Moreover, as the world is becoming interconnected enabling formation of new organizational structures and alliances internationally, leveraging upon the insights developed from global information resources (e.g. SM), and management of such information resources is pivotal to survive and succeed in the global marketplace (Rialp-Criado \& Rialp, 2020).

Social media-driven big data (hereafter 'SM data') are an important source of unstructured and external data that are suboptimally utilized for CI purposes (Xu et al., 2017). Facebook alone has more than 2 billion active users (including businesses), 1.5 billion of whom are active on a daily basis (Marr, 2018). Other SM platforms such as Twitter, Instagram, WeChat, and Snapchat contribute to the creation of staggering volumes of data comprising transactions, communications, and information. The SM platforms being used globally are becoming potent IT infrastructure for the companies to expand internationally (Rialp-Criado \& Rialp, 2020). This is seen from the impact of SM, which is visible at, both, global (e.g., Rialp-Criado \& Rialp, 2020) as well as national cultural level (e.g. Halawani et al., 2020's study conducted in a Lebanese context). The insights derived from SM data are helping companies understand local and global market sentiments and strategize accordingly (Iftikhar \& Khan, 2020). Therefore, SM analytics (SMA), or the collection and analysis of SM data to create business value, provides a significant opportunity to acquire CI to improve business operations and competitive positioning (Halawani et al., 2020). This position is in line Chen and Ching's (2004) study as they suggest that organizations should use technology infrastructure to improve their CI capabilities for customer relationship management and product innovations.

However, the academic understanding of the role of SMA in obtaining CI is fragmentary and limited. This study addresses this gap by posing the following research question: What role does $S M$ analytics play in providing CI beyond traditional competitor-based insights? The motivation for this research question stems from a number of issues that need attention. First, current SM-driven CI research (e.g., He et al., 2015; Xiang et al., 2017) has mainly focused on customer sentiment text analysis or, to some extent, competitor analytics, CI, however, is much broader in scope, going beyond the traditional customer-competitor orientation (Porter, 1980). Köseoglu et al. (2019) explain this important consideration:

The scope of the CI process is broad since it is directly related to both organizational strategy and strategic decision-making . . . Data about CI activities comes from both external and internal environments such as customers, the market, competitors, and business interactions. (p. 284)

This conceptualization is in line with the findings of a large-scale survey on CI by Calof (2017), confirming the broad scope of CI. Guimaraes, Sato and Kitanaka (1999) concur as they found that CI scope is wider and includes implementing business change. Second, many current studies on acquiring CI from SM are preliminary in nature, such as developing a conceptual framework (Rathore et al., 2016), presenting a consolidated review of the literature (Lee, 2018), or developing an analytical approach to using SM for CI (He et al., 2017). Third, despite the relative novelty and developing nature of SMA for CI, apart from a few examples (e.g., Köseoglu et al., 2019; Muninger et al., 2019), few studies have taken a theoretically informed qualitative approach to building a robust and in-depth perspective on the subject. 
We argue that these issues limit the applicability of CI in seeking broader insights into factors beyond customers and competitors. This necessitates further work to develop a richer understanding of CI advancing work by earlier studies (Calof, 2017; Guimaraes, Sato \& Kitanaka, 1999; Köseoglu et al., 2019; Porter, 1980). Moreover, the role of SMA in enabling improvements in operational capabilities such as demand prediction, supply chain and business performance as have been highlighted in recent studies (e.g., Halawani et al., 2020; Iftikhar \& Khan, 2020) further necessitates building knowledge on the contributions of SMA in CI. The study achieves this objective.

\section{LITERATURE REVIEW}

\section{Theoretical Underpinning: Competitive Intelligence Theory}

CI has its roots in strategic planning and management (Prescott, 1995). Although the concept of CI originally focused on competitor analysis, it has since evolved to become much broader (Safarnia et al., 2011). CI can provide insights about various strategic and tactical options for economic and technical decisions in organizations (Calof et al., 2016). Thus, CI includes "competitor intelligence as well as intelligence collected on customers, suppliers, technologies, environments, or potential business relationships" (Dishman \& Calof, 2008, p. 768).

Cleland and King (1975) developed the following eight premises of CI theory:

1. CI is essential for successfully competing in a market by providing an understanding of markets, competitors, and customers, which is pivotal for strategy formulation and implementation to achieve a strategic advantage (Safarnia et al., 2011).

2. Market dynamics necessitate adequate systems for collecting CI. This premise is based on the understanding that customers' changing needs and demands, supply variations, and other market forces necessitate a system that can provide insights for companies to continually adjust their operations and offerings (Prescott, 1995).

3. Companies require total systems for providing effective CI.

4. CI collection should be both rigorous and personalized.

5. CI should be action oriented, providing exception-based information for managerial decision-making.

6. CI can be gathered from a variety of sources, some of which may appear to be unprofitable.

7. CI should be considered bidirectional.

8. CI can be effectively collected without resorting to illegal means.

Building on these tenets of CI theory and drawing upon Calof's (2017) conceptualization, we go beyond the traditional scope of competitor analysis by including the dimensions of sales and business development and product development in our investigation of SMA-driven CI. The inclusion of these two additional dimensions was guided by the empirical findings of Calof (2017), who concluded that among the various dimensions, sales and business development and product development are the main focus of companies in collecting CI. Moreover, SM is an emerging technology in sales and product development; thus, focusing on these two areas in examining the role of SMA in obtaining CI aligns with the objectives of this study.

\section{The Role of Social Media Analytics in Competitive Intelligence}

Our literature review shows that one of the primary reasons for obtaining CI is product and service innovation. Rathore et al. (2016) argue that SMA can help improve product design and facilitate new product development. For instance, comments in SM forums about product defects may be used to incorporate new features during product development. In addition, through sentiment analysis of SM content (e.g., tweets), companies can gain intelligence by comparing the perceived product value 
of their own and their competitors' products, enhancing business value and market intelligence capabilities (He et al., 2015).

New product development requires an understanding of customers' behaviors, needs, and concerns (Rathore et al., 2016). Thus, a number of studies have examined the role of SMA in gaining CI in relation to customer issues (e.g. He et al., 2016). SM data can provide intelligence on customer sentiments, preferences, and tastes, product and service function and feature requirements, and future development needs, facilitating planning for daily and future operations (He et al., 2015; Xu et al., 2017). While tweets have word limits, online reviews with greater text flexibility can provide firms with greater insights about customer sentiments, purchase intentions, and opinions about competitors' products and services.

Xu et al. (2017) used text mining to analyze customer reviews of hotels. However, caution should be exercised when using reviews for decision-making processes because the quality of reviews can vary (Xiang et al., 2017). Thus, Chang et al. (2019) proposed an integrated approach involving elements such as data crawlers, data preprocessing, sentiment-sensitive tree construction, and visual analytics to overcome the algorithm limitations for gaining decision-making information.

SM can also be used to build relationships with customers, improve decision-making, and address customer complaints. The provision of location-based services and integration of product features according to regional and cultural variations will help companies enlarge their customer portfolios (He et al., 2017). However, the real-time and around-the-clock nature of SM input makes it challenging to stay current with changing sentiments; thus, more research is required to find practical solutions.

The literature suggests that SMA can be used to identify areas in which a company is lagging compared with its competitors (He et al., 2015). For instance, using sentiment analysis, opinion mining, and statistical analysis of tweets following the release of iPhone 6, Kim et al. (2016) found that the number of tweets containing the words "iPhone 6" or "Galaxy S5" reflected the market leadership of the respective companies and, to some extent, customers' purchase intentions. Thus, CI obtained from an analysis of competitors' SM presence can be used to understand new trends, design new products, and develop marketing strategies to enlarge a company's customer portfolio (He et al., 2017). However, given that the effect of SM has been found to vary, even within the same business segment (He et al., 2016), it is vital to investigate the manner in which SM is leveraged by firms to gain benefits.

The key to achieving operational benefits is to build an effective information flow through which to engage with customers and keep them about the company's progress, such as updates on product or service offerings (Lee, 2018). Sheng et al. (2017) argue that analysis of big data, including SM data, can provide a broader view of the effects of information flow on business operations in real time. Additionally, SMA was found to help companies obtain information about customers to develop strategies for new products and/or personalized services.

The above review shows that while studies have explored the use of SMA for the traditional understanding of customer needs, market sentiments, and competitors, the large volume and variety of SM data can provide insights beyond traditional knowledge, including costs and supply chain management. However, little research has examined the role of SMA beyond the traditional scope, resulting in a gap in the knowledge on the various types of CI that can be obtained from SMA. Therefore, this study fills this gap by taking a theoretically informed approach to examining the role of SMA in providing CI from a multidimensional perspective.

\section{RESEARCH METHODOLOGY}

\section{Research Design and Sample Selection}

Given that SM is a relatively new field of research and studies on the use of SMA for obtaining CI are limited, this study used an exploratory qualitative research methodology. Data were collected using 


\begin{tabular}{|c|c|c|c|c|}
\hline Respondent No. & Job Title & Organization type & Organization size & Industry \\
\hline Re spondent 1 & Сто & Non-profit & Large & $\begin{array}{l}\text { Non-profit business incubator } \\
\text { services }\end{array}$ \\
\hline Re spondent 2 & Investment VP & Investment & Small & Investment services \\
\hline Re spondent 3 & IT department staff & Retailing & Medium & Product retailing \\
\hline Re spondent 4 & IT department staff & Manuf acturing & Large & Product m anuf acturing \\
\hline Re spondent 5 & Data Scientist & Social Media & Large & Social me dia services \\
\hline Re spondent 6 & Investment Analyst & Inte met & Small & IT services \\
\hline Re spondent 7 & IT manager & Manuf acturing & Large & Product m anuf acturing \\
\hline Re spondent 8 & VP & E-business & Large & Product retailing \\
\hline Re spondent 9 & Data Analyst & Telecommunication & Large & Telecom services \\
\hline Re spondent 10 & Sales Analyst & Logistics & Medium & Logistics services \\
\hline Re spondent 11 & IT staff & Manuf acturing & Large & Product m anuf acturing \\
\hline Re spondent 12 & Marketing manager & Manufacturing & Large & Product m anuf acturing \\
\hline Re spondent 13 & Data Analyst & Manuf acturing & Large & Product manufacturing \\
\hline Re spondent 14 & IT manager & Manuf acturing & Medium & Product m anuf acturing \\
\hline Re spondent 15 & Marketing manager & Manufacturing & Medium & Product manuf acturing \\
\hline Re spondent 16 & IT departmetn staff & Non-profit & Large & $\begin{array}{l}\text { Non-prof it business incubator } \\
\text { services }\end{array}$ \\
\hline Re spondent 17 & IT manager & Service & Medium & Product manufacturing \\
\hline Re spondent 18 & Sales analyst & Service & Medium & Product m anuf acturing \\
\hline Respondent 19 & IT departmetn staff & Inte met/eCommerce & Large & In ternet products and services \\
\hline Re spondent 20 & IT consultant & Inform ation Tech nology & Large & IT products and services \\
\hline
\end{tabular}

semi-structured interviews, enabling the researcher to seek a more detailed and in-depth understanding of the issues under investigation.

A purposive sampling technique was used to identify respondents who could provide an in-depth understanding of the various aspects of the role of SMA in gaining CI (Patton, 1990). This approach is consistent with those of earlier SM studies (Muninger et al., 2019). One of the authors of this paper identified respondents through her links with the SM and manufacturing industries and from Weibo. These respondents were knowledgeable and had been involved in business intelligence, big data, or SM-related projects. Their credentials were checked when seeking consent to participate in the interviews. We conducted 20 semi-structured interviews following recommendations of Morse (2000) and Gruber et al. (2008), who posit that 20 interviews are sufficient for qualitative research, and Köseoglu et al. (2019), who assert that a minimum of 15 interviews is ideal. Respondents included information technology managers, chief technology officers, data analysts, data scientists, and marketing managers. Sixty percent of respondents came from large organizations, $30 \%$ from medium-sized organizations, and the remaining 10\% from small organizations (see Table 1 for details on respondent profiles).

A semi-structured questionnaire was developed based on the literature review, confirming to works by Calof (2017) and Köseoglu et al. (2019), and the abovementioned tenets of CI theory. The questionnaire consisted of two parts: the first comprised questions to collect respondent profile information, and the second comprised questions related to CI (see Table 2).

\section{Data Collection and Analysis}

Data were collected from 20 semi-structured interviews with industry experts (see Table 1). Being a qualitative study, the sample size was adequate and consistent with earlier studies (e.g., Muninger et al., 2019; Schlagwein \& Hu, 2017). Moreover, given that the research on SM is still in the early stages of development, a sample size of 20 was sufficient to meet the purpose of developing basic knowledge. 
Table 2. Item measures used in the semi-structured questionnaire

\begin{tabular}{|c|c|c|c|}
\hline $\begin{array}{l}\text { Key issues identified } \\
\text { from literature which } \\
\text { were adopted for } \\
\text { building semi-structured } \\
\text { open-ended questions }\end{array}$ & $\begin{array}{l}\text { Areas of competitive Intelligence } \\
\text { concept as per Calof, (2017); } \\
\text { Köseoglu et al. (2018) }\end{array}$ & Open ended questions & $\begin{array}{l}\text { Some literature } \\
\text { References }\end{array}$ \\
\hline 1. Cost & Business Development & $\begin{array}{l}\text { In your opinion, do } \\
\text { analytics using data } \\
\text { from social media can } \\
\text { help to understand cost } \\
\text { drivers? }\end{array}$ & $\begin{array}{l}\text { Štefániková and } \\
\text { Masàrovà, (2014) }\end{array}$ \\
\hline 2. Location-based services & $\begin{array}{l}\text { Product Development/Business } \\
\text { Strategy }\end{array}$ & $\begin{array}{l}\text { In your opinion, do } \\
\text { analytics using data } \\
\text { from social media can } \\
\text { help to provide location } \\
\text { based service? }\end{array}$ & $\begin{array}{l}\text { Fan, Lau and Zhao } \\
\text { (2015); Hu et al. } \\
\text { (2019) }\end{array}$ \\
\hline $\begin{array}{l}\text { 3. Supply chain } \\
\text { management }\end{array}$ & Sales or Business Development & $\begin{array}{l}\text { In your opinion, do } \\
\text { analytics using data } \\
\text { from social media can } \\
\text { help to improve supply } \\
\text { chain management? }\end{array}$ & $\begin{array}{l}\text { Fan, Lau and Zhao } \\
\text { (2015); Hu et al. } \\
\text { (2019) }\end{array}$ \\
\hline 4. Information flows & $\begin{array}{l}\text { Business Development/ } \\
\text { communication }\end{array}$ & $\begin{array}{l}\text { In your opinion, do } \\
\text { analytics using data } \\
\text { from social media } \\
\text { can help to identify } \\
\text { bottlenecks in } \\
\text { company's information } \\
\text { flows? }\end{array}$ & $\begin{array}{l}\text { Fan, Lau and Zhao } \\
\text { (2015); Hu et al. } \\
\text { (2019) }\end{array}$ \\
\hline
\end{tabular}

Interviews were conducted face-to-face or online through Skype or SM communication tools such as WeChat. On average, interviews lasted 45-60 minutes. Some were audio recorded with interviewee consent, while others were recorded by note-taking. All interviews were conducted by one author to ensure consistency and reliability in the data collection process. Because the research was conducted in China, all respondents spoke Chinese during interviews. The author who collected the data was bilingual in Chinese and English and translated the Chinese transcripts into English for analysis purposes.

The study adopted an open coding approach to code the interview data using NVivo 12.0 software, which is in line with prior studies in the SM domain (Muninger et al., 2019). First, data were checked to ensure completeness. Because all respondents answered all questions, a high level of quality was achieved for the compare and contrast stage of analysis. Initial data analysis was theoretically driven and drew upon questions borrowed from previous studies (see Table 2) to code the data into main themes (Strauss \& Corbin, 1994). Next, the answers within each theme were inductively analyzed, compared, and contrasted to examine similarities, differences, and patterns in responses to identify subthemes and code data accordingly.

The following example illustrates the coding process: Based on the results of the questionnaire, one theme was labelled "Improving supply chain management". All 20 respondent answers for this theme were carefully analyzed, resulting in the identification of various subthemes, coded as "Understanding of supply and demand, demand planning, and inventory management", "Identification of factors and uncertainties that may affect the supply chain", and "Limitations and recommendations". The analysis of data in this manner is also consistent with that of other studies in the SM context (e.g., Köseoglu 
Table 3. Themes and subthemes extracted from NVivo

\begin{tabular}{|c|c|c|}
\hline Themes, Sub-themes codings of data extracted from NVivo 12.0 & Sources & Quotes \\
\hline I. Understanding of sources of costs & 12 & 12 \\
\hline Limitation (information sufficiency, utility of SM, and technological availability) & 9 & 9 \\
\hline Use internal data or combine with internal data for costs & 4 & 4 \\
\hline Viability and nature of sources of costs & 1 & 1 \\
\hline II. Providing location-based services & 17 & 17 \\
\hline Events identification and Other Opportunities & 5 & 5 \\
\hline Limitations (privacy, regulatory compliance) and recommendations & 5 & 5 \\
\hline Develop privacy preserving tools & 1 & 1 \\
\hline Increased people mobility may lessen utility of SM for location-based services & 1 & 1 \\
\hline Localization strategies & 4 & 4 \\
\hline III. Improving supply chain management & 16 & 16 \\
\hline Identification of factors and uncertainties that may affect the supply chain & 2 & 2 \\
\hline Limitations and recommendations & 4 & 4 \\
\hline Combine SM data with other data & 3 & 3 \\
\hline $\begin{array}{l}\text { Understanding of supply and demand, demand planning, and inventory } \\
\text { management }\end{array}$ & 6 & 6 \\
\hline IV. Identifying bottlenecks in information flow & 11 & 11 \\
\hline Limitations and recommendations & 8 & 8 \\
\hline Use internal data or combine with SM data for info flow effectiveness & 2 & 2 \\
\hline Understanding of weaknesses of effectiveness of info flow and opportunities & 5 & 5 \\
\hline Opportunities of finding new directions & 2 & 2 \\
\hline
\end{tabular}

et al., 2019). Once the data were coded into themes and subthemes, responses were systematically combined to enable interpretation of the data. Details of themes and subthemes are provided in Table 3.

We also analyzed the coded data using coding queries and matrix coding queries in NVivo to identify the association between respondent attributes (e.g. job title, type of organization, and organizational size) and responses. Using matrix coding queries, we could gain insights into perspectives according to respondent profile, enabling us to consider the possible reasons for these unique perspectives.

\section{Reliability and Validity}

A number of steps were taken to ensure reliability and validity. First, given that the topic under study has had limited research, methodological coherence between the study aims (exploratory) and research method was ensured by taking a theoretically informed approach, collecting data from semi-structured qualitative interviews (which is aligned with exploratory investigations), and analyzing data by coding them into themes and subthemes to identify factors related to the role of SMA in obtaining CI. Second, item measures used in developing the research questionnaire were drawn from prior studies on SM and confirming to conceptualization in Calof, (2017) and Köseoglu et al., (2019) (see Table 2). Third, care was taken to choose respondents with knowledge on the subject by confirming whether they had been involved in SM, big data, or business intelligence. Fourth, the use of NVivo for data coding and analysis (through queries and matrix queries) allowed systematic, granular, and replicable 
coding and analysis, contributing to the reliability of findings (Lincoln \& Guba, 1985). Finally, the data were coded by two authors independently. The level of agreement between both manual and NVivo-based coding, which were conducted independently of each other, was $100 \%$, demonstrating high intercoder reliability. For example, using manual coding, data from one of the questions were coded into the theme "Improving supply chain management" and subthemes, "Improved demand planning and inventory management", "Uncertainty identification", and "Combination of data with other data to support decision-making". This manual coding was fully consistent with the coding for the same theme using NVivo (see Table 3 for NVivo coding).

\section{RESULTS AND DISCUSSION}

Analysis of the interview data offered several new insights and identified limitations in the role of SMA in obtaining CI. These are discussed in detail below and presented in Table 4.

\section{Understanding Sources of Costs}

The data analysis shows that there were mixed views about the role of SM data in providing insights about the sources of costs. Obtaining a granular understanding of a company's costs in comparison with its competitors may be an area in which SM data can provide insights, According to Respondent 2:

The profit goes down if cost is larger than income. However, why is the increase in cost larger than the increase in income? This can be analyzed through big data analytics on social media data. Is it because of product transition? Or is it because of fierce competition? Big data will help to find the possible reasons and take corresponding actions to cut cost.

This finding indicates the potential for examining SM data to identify cost drivers. We believe that user-generated content (e.g. opinions, comments, feedback, blogs, or customer interactions) can provide insights about cost differentiation (e.g., delivery, troubleshooting, customer service, and maintenance costs). This understanding may help companies identify whether costs in some areas are higher or lower than those of their competitors. These costs may increase or reduce operational, and ultimately product and service, costs. This finding expands on those of earlier works (e.g., Štefániková \& Masàrovà, 2014) on the role of SMA in providing insights on costs.

\section{NVivo Query Results Analysis}

Matrix coding analysis showed that respondents from manufacturing, service, and large companies were uncertain about the role of SMA in providing insights on costs and recommended that internal financial data (potentially in combination with SMA data) be used to gain such insights. Such a finding seems reasonable given that SM-driven information about costs may be broad and unfocused, making it difficult to gain valid insights. To overcome this challenge, data analysts and other relevant employees should work together to design appropriate algorithms for mining actionable CI from the large SM data pool.

\section{Providing Location-Based Services}

The results show that SMA can help provide location-based services, increasing the ability to be competitive, particularly given the increased level of population mobility. Users may be categories based on location logs to further analyze similarities in habits and tastes. Businesses operating in countries with vast territories (e.g., China) often need to customize their offerings and marketing because people living in different geographical regions have different customs and preferences. Such information can help develop location-based marketing strategies in terms of, for example, promotional messages, pricing, and product packaging. Respondent 9 explained that "people living in the same 
area tend to have similar customs, habits, tastes, and preferences. These are valuable information to develop [a] location-based marketing strategy."

Combining SM users' locations with their interactions, discussions, and transactions can provide intelligence on customers' purchasing behaviors, intentions, and future activities. This knowledge may be used to refine existing and design new localized sales and marketing strategies, ultimately leading to increased customer satisfaction. Further, mining the SM location log may not only provide insights into the company's own products and services but also yield valuable intelligence on competitors' offerings, helping to localize the company's offerings. Particularly, as SM is a globally used technology, it can be very useful in helping companies in their internationalization endeavors by providing an understanding of customers' needs based on customers' geographical locations. Such an understanding will enable tailoring sales and marketing campaigns to the geographical locations and help company expand internationally. This is in line with findings of Rialp-Criado and Rialp's (2020) study who argued that speed of use of SM is positively associated with the speed of internationalization.

Another perspective that emerged from the analysis is that while regional events may influence the local market, they may not be prominent enough to be covered by the mainstream media. SMA for a particular location may help identify events being discussed by SM users that are not promoted through mainstream information channels. The intelligence gathered can then be used for sales, marketing, and customer engagement activities, enhancing the competitive position of a company:

For example, social media is always a good place to know about local events. These events may have an impact on the local market but may not be broadcast on [the] news. If log data is included in big data analytics, companies may find these events in time and make adjusted response to these local events. (Respondent 16)

\section{NVivo Query Results Analysis}

The query results show that respondents from both the manufacturing and service sectors were enthusiastic about the use of SMA for gaining location-based CI to design and refine their business strategies. Further, we found that regardless of organization size, all respondents considered SMA to be useful for gaining location-specific CI. However, large companies were more concerned about privacy issues, which is understandable given that large companies face bigger legal, financial, and reputational risks.

\section{Improving Supply Chain Management}

The results show that SMA can improve at least three aspects of supply chain management. The first aspect is demand planning and inventory management. SMA can help identify customers' preferred products and services that are more likely to be purchased or consumed. This information may be used to support demand planning and inventory management to improve supply chain activities, as explained by Respondent 2: "It can provide a sign ahead of time before the transaction records are available. Good comments on products tend to attract more purchase behavior, which can be observed on social media analytics."

The second aspect that emerged from our analysis is that the real-time capture of SM data can help identify uncertainties that may affect supply chain operations. SM data such as group discussion threads, blogs, comments, and product reviews can yield abundant information about sentiments, events, or factors that may affect supply chain processes in the near future. Respondent 20 explained this succinctly: "I guess there are many factors [that] can influence the supply chain management process, and big data analytics is able to detect these factors and uncertainties."

The third aspect that emerged from the interviews is that SM data should be combined with data from other sources such as transactions to gain a more complete picture of supply chain management: "I think it will be more effective if combined with other data such as logistic data or transaction data" 
(Respondent 18). This important finding advances that of an earlier study, which found that analysis of customer sentiments in Twitter helped improve supply chain efficiency (Singh et al., 2018). Chae (2015) also analyzed Twitter, agreeing that SM plays an important role in supply chain practices such as demand shaping, supply chain risk management, and stakeholder engagement. Our findings also further strengthen work of Iftikhar and Khan (2020) who demonstrated through a case study that SMA help improve demand forecasting accuracy in a supply chain. While it is known that SMA can help build supply chain management capabilities for logistics, innovation, and service recovery (Singh et al., 2018), more research is required to understand the role of SMA in supply chain management. Algorithms and technologies available for analytics are also in the early stages of development. Therefore, using other supply chain data together with SM data may be a more effective approach for companies to understand the benefits of and build confidence in using SM data to improve supply chain effectiveness.

\section{NVivo Query Results Analysis}

Our query analysis shows that mostly respondents from large and medium-sized companies considered SMA a valuable source of CI for supply chain management, particularly for demand planning and uncertainties. This may be because opinions, comments, and discussions on SM can provide vital intelligence about the overall sentiments of consumers, which can help in demand planning, inventory management, and adjustments in overall supply chain management strategies. Moreover, the continuous generation of SM data provides the opportunity to conduct SMA in real time or over shorter periods, which may be of enormous benefit to supply chain management adjustments and potentially increase profits. Interestingly, we found no observable patterns between the views of respondents belonging to manufacturing versus service or other types of companies. This could be because the use of SM data for supply chain management is still new and not sufficiently streamlined.

\section{Identifying Bottlenecks in Information Flow}

Our results show that SMA can provide a better understanding of information flow and help identify bottlenecks. Bottlenecks in information flow arise from issues such as the sharing of incomplete, inaccurate, biased, or delayed information and companies being unable to reach out to desired customers and stakeholders. An understanding of bottlenecks can help companies adjust their communication strategies and improve the fluency of their information flow, which can enhance their competitive abilities:

I think most of the companies use social media as a tool to communicate with consumers and also use it as a channel to post company information. Therefore, big data analytics on social media data can find out how the information flows and also find out the bottlenecks in information transformation. (Respondent 15)

SMA can provide insights on weaknesses in the management of a company's information flow. Companies can also learn about the effectiveness of their information flow compared with that of their competitors from users' comments and opinions in SM. This intelligence may be exploited to improve communication and find new means of communication, ultimately improving customer engagement, sales, and profitability (Respondents 2, 4, and 5).

\section{NVivo Query Results Analysis}

Our query analysis shows that using SM data to identify bottlenecks in information flow is contentious. Respondents from large and manufacturing companies saw the value in using SMA for gaining insights into information flow effectiveness but also believed that combining SM data with other data would be more effective. Query results suggest that business-related respondents (e.g., marketing and sales) 
were more enthusiastic about using SMA for gaining insights into information flow, while technical staff (e.g. information and technology managers) advised caution in relying on SMA for this purpose.

\section{Limitations of Social Media Analytics in Obtaining Competitive Intelligence}

Our analysis shows that despite the seemingly many benefits of using SMA for obtaining CI, the insights gained from SMA also have many limitations. One argument that clearly emerged from the study findings is that SM data should be combined with companies' internal financial data to identify sources of costs for implementing strategies to improve cost competitiveness, as explained by Respondent 17: "It is better to use social media data together with some internal financial data to give an unbiased view."

Further, our analysis shows that privacy concerns and related regulations are challenges to providing location-based services. Our results suggest that companies should be cautious in handling location-based data because users may be concerned about privacy violations. One suggestion was that technology development should focus on building privacy-preserving algorithms that can provide companies with desired intelligence while preserving the privacy of users.

Moreover, Respondents 1, 3, 6, 7, 8, 14, 17, and 18 pointed out the limitations of using SM data for gathering intelligence on information flow, including (1) that it may be effective for some industries but not others (Respondents 3 and 7); (2) the variability in dependency on the use of SM for the company's business (Respondent 8); and (3) that SM data alone are insufficient for obtaining a full understanding of the effectiveness and bottlenecks in information flow. Respondent 1 recommended using SM along with internal data: "The bottlenecks in company information flows should be analyzed within the company with internal data and internal business process. The external data from social media may have little influence on this."

These results underline the importance of having a strategy that considers the strengths and weaknesses of SMA-driven CI and leverages the benefits of using SMA for CI.

\section{DISCUSSION}

SMA can obtain multidimensional aspects of CI, confirming the tenets of CI theory and the conceptualizations of Calof (2017) and Köseoglu et al. (2019). While earlier works (e.g., Štefániková \& Masàrovà, 2014) have shown that CI can help reduce costs, the present study provides a new perspective that SMA can provide CI to understand sources of costs. This knowledge then be used to make informed decisions on cost management and improve cost competitiveness. Sources of costs can also be better understood by gathering location-specific CI. Earlier works (e.g., Lee, 2018) have discussed the possibilities and need for location-specific analytics using SM. This study provides further evidence that SMA can provide intelligence on location-specific habits, tastes, preferences, and current and future purchasing intentions, which can be used for improved decision-making. SM being a technology that is used across various cultures and nationalities (e.g., Wechat in China or Whatsapp globally), our results extend work by earlier studies (e.g. Halawani et al., 2020; Iftikhar \& Khan, 2020; Rialp-Criado \& Rialp, 2020) which have emphasized managing SM as a key information resource for achieving operational performance and sustainable growth.

SMA appears useful for providing location-specific information. These results strengthen earlier work where Balduini et al. (2012) underlined the usefulness of SM data to provide personalized and localized services. As well, it can help in increased internationalization and global reach-out as is argued by Rialp-Criado and Rialp (2020). Extending the discussion, Brandt et al. (2017) found that spatial and semantic analytics of SM data can provide useful insights for smart tourism management. However, the continuous nature of data generation through SM may make it challenging to keep up with the analysis of data and tailor products in a timely manner. Tailoring products/services, sales, and marketing strategies for short periods based on location-specific intelligence seems a more realistic approach. 
Our analysis also shows that SMA-driven CI can help in achieving supply chain and information management efficiency. From an IT perspective, SM provides multi-format big data about customer sentiments, supply and demand variations, and environmental factors that effect supply chain management. Analytics of such data has a positive impact on supply chain and organizational performance (Iftikhar \& Khan, 2020). Further, real-time around-the-clock data gathered from a variety of SM channels can help in understanding market dynamics and customer needs, the analysis of which may facilitate with addressing supply-demand uncertainties and achieving supply chain efficiency (Singh et al., 2018).

Moreover, we find that SMA provides insights on effectiveness of information flow, which facilitates improved interactions with customers, suppliers and other stakeholders, as also proposed by Bukhari et al. (2010). However, we believe that optimizing the potential value of SM and associated analytics requires technological advancements, policy and regulatory infrastructure development, and education of user communities. The current level of knowledge shows that SMA is still in the early stages of development, and extensive efforts are required to gain the full benefits. In particular, technological advancements in algorithm development, real-time analytics, visualization, and privacypreserving data analytic tools, hardware, and software are needed to crunch multiformat big SM data for achieving business efficiency. One suggestion from our analysis was the need to develop privacy-preserving algorithms.

\section{CONCLUSION}

The exponential growth of SM is changing the business environment, and companies are using SM as a vital source of CI to understand competition dynamics and implement appropriate strategies to remain sustainable in the marketplace. Advancing the knowledge on this subject, this study has concluded that SMA plays a valuable role in obtaining CI. Complementing earlier works, the results offer multiple new insights into the benefits and limitations of SMA in obtaining CI, presented in Table 4, and discussed as follows:

1. Sources of costs: Companies can identify sources and benchmarks of some types of costs (e.g. delivery, troubleshooting, customer service, and maintenance costs) against comparable/ competing businesses based on SMA. However, this intelligence is limited in nature and should be complemented with internal cost data to obtain more valuable insights and improve cost competitiveness.

2. Location-based tailored products and services: The multilayered (local, regional, and global) nature of SM data enables the gathering of intelligence on upcoming location-specific events that may not be publicized in mainstream media. This intelligence creates business opportunities to tailor sales and product development and marketing strategies as well as expand globally (RialpCriado \& Rialp, 2020).

3. Supply chain management effectiveness: Using SMA-driven CI, companies can achieve enhanced demand planning and inventory management by identifying consumers' preferences or positive views about certain products - a finding which advances earlier work by Iftikhar and Khan (2020). Other areas in which SMA may facilitate supply chain management include: (1) understanding the uncertainties that may affect supply chains based on real-time data capture (e.g., from group discussion threads, blogs, comments, and product reviews); and (2) achieving better visibility of the supply chain by combining supply chain-related data (e.g., logistics data) with SM data. However, algorithms and technologies for the analysis of SM and supply chain data to provide useful CI need to be developed.

4. Information/communication diffusion: To some extent, companies can gain insights on the effectiveness (e.g., timely delivery, ability to reach out to or communicate with the target audience, and message format, completeness, correctness, currency, and accuracy) and bottlenecks 
Table 4. Results summary

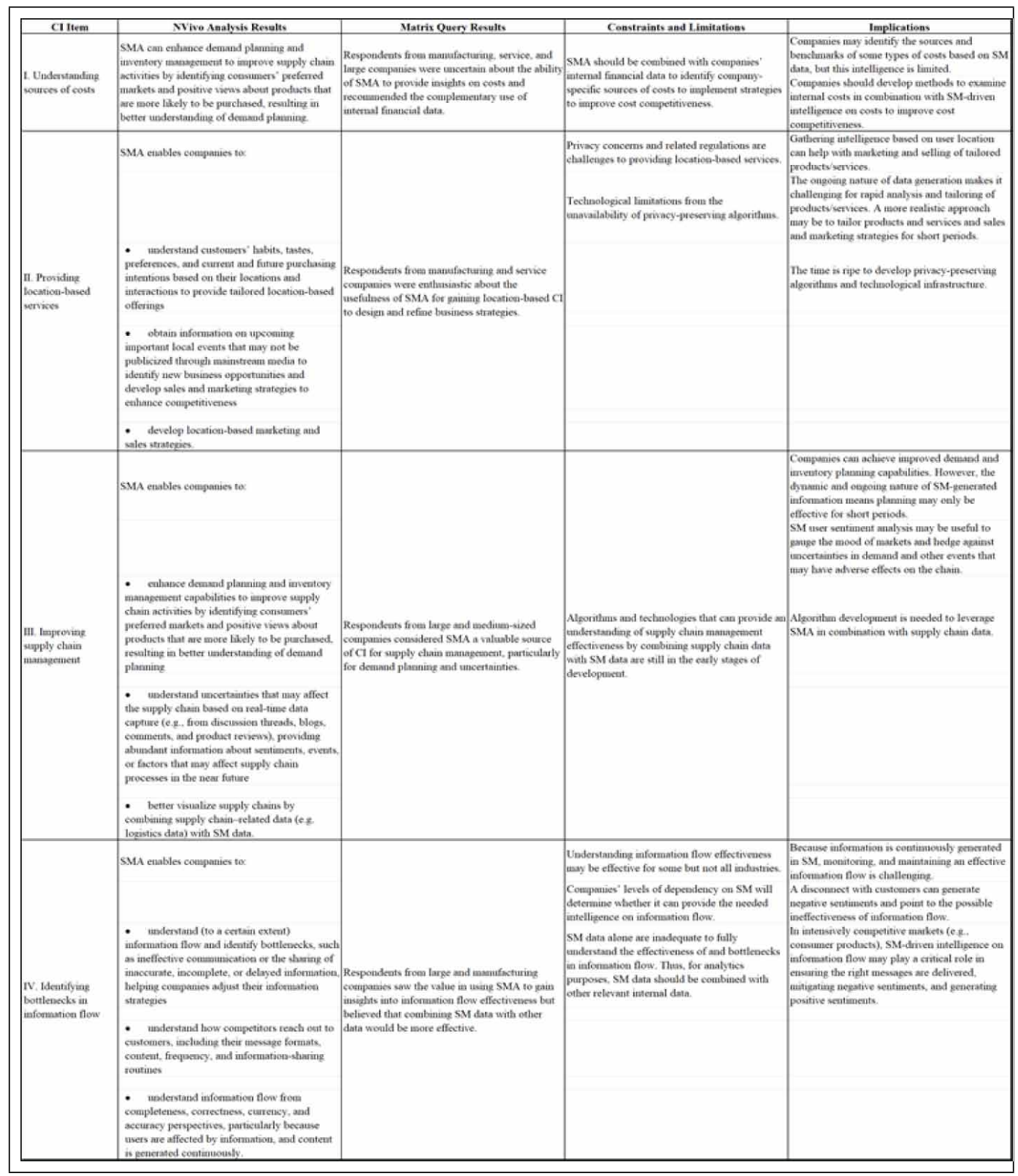

Notes: Cl: competitive intelligence; SM: social media; SMA: social media analytics.

in information flow. Our results show that SM data alone are inadequate to understand the effectiveness of or bottlenecks in the information flow and should be combined with other relevant internal information to gain better intelligence on information flow.

To conclude, SM data are a vital source of CI to enhance internal efficiencies and external positioning. The multipronged effects of SMA may empower organizations to effectively manage a range of elements, including supply chains, information flow, personalized offerings, and location- 
based provisions. There could be variations in the way SM is used across different cultural contexts (such as Wechat is the main SM app used in China versus Whatsapp is favored outside China), but it seems that the management and use of information driven from SM for business have similarity of purpose and benefit proposition (Halawani et al., 2020). Further, we argue that while the results of this study done in a Chinese context correspond to and extend earlier work (e.g., Halawani et al., 2020; Iftikhar \& Khan, 2020), the understanding of complementarities in the role of SMA for CI across various cultural contexts need further examination.

It is also pertinent to note that organizations cannot solely rely on SMA. SM is an open medium in which every actor has an equal opportunity to gain the desired CI, making it difficult to achieve a long-term sustainable advantage based on SMA alone. Thus, organizations also need to guard against leakage of information to competitors through SM channels. Therefore, it is beneficial to complement SMA with company-specific operational, financial, and managerial data to achieve the desired results.

Moreover, because a large proportion of SM users comprise members of younger generations, SMA may generate skewed results with limited applicability to other tiers of society. The cost, time, and effort involved in collecting and analyzing SM data must be weighed against its potential benefits. Although SM is popular, it is not suited to every type of business; thus, its limitations and constraints must be considered.

\section{IMPLICATIONS, LIMITATIONS, AND FUTURE DIRECTIONS}

\section{Implications for Theory and Practice}

This study makes several contributions to theory and practice. The study contributes to global IT information management literature by developing insights on the role of SMA in providing multifaceted information to organization in the form of CI (particularly as SM is a globally used technology). We show that SMA can provide expanded knowledge resources to businesses, including insights on information flow effectiveness, supply chain management, and costs, beyond the traditional scope of $\mathrm{CI}$ (which is predominantly focused on competitor analysis), as proposed in prior studies. These results show that $\mathrm{CI}$ is a multidimensional construct, contributing to an improved theoretical measure of CI than that limited to competitor analysis. Such an understanding will advance theoretical knowledge development on the strategies for management of information resources with a particular focus on SM and other related communication technologies. The Chinese context of the study will also help in advancing theoretical knowledge on the cultural and nationalistic perspectives of SM use for business as well.

The study also shows that the behaviors of competitors, customers, suppliers, and other stakeholders may be understood through analysis of SM-based information. While showing the usefulness of CI theory for examining SM-related issues, the results complement and extend those of earlier studies (e.g., Halawani et al., 2020; Iftikhar \& Khan, 2020; Rialp-Criado \& Rialp, 2020) and contribute to the research on the development of SM theory in global information management context. Finally, our results have identified some benefits and limitations of using SMA to obtain CI, as presented in our framework (see Table 4), adding to the theoretical understanding of the role of SMA.

The results provide insights for chief information officers, SM managers, and operations managers about the role of SMA in providing CI, guiding them to plan and implement business and technological strategies to leverage SM-driven global information resources for enhanced decisionmaking and operational efficiencies. The results will help managers to put in place strategies and take appropriate actions for efficient collection and processing of SM driven information to identify insights for competitive advantage. Guided by findings of the study, managers will be able to recognize the opportunities (such as for improved supply chain performance, cost efficiencies, and tailored sales and marketing management) available at a global as well as national level by leveraging SMdriven information. Such a recognition will enable developing plans to use SM-based information 
resources effectively for business benefits. The limitations and benefits identified in this study (see Table 4) serve as a framework for considering the necessary improvements in SMA algorithms and technologies to maximize the benefits of using SMA for business purposes.

\section{Limitations and Future Directions}

This study has some limitations. First, while rich in outcomes and knowledge, like other qualitative studies, the findings of this study are limited by the subjective opinions of field experts. Second, although our sample size was adequate and consistent with prior studies, the number of people interviewed in this research was limited. Third, interviews were conducted in one country; therefore, the generalization of findings to a broader context and other business environments may also be limited.

This study opens a number of avenues for further research. For example, a qualitative study with a larger sample size comprising individuals from several countries will help consolidate the CI construct and add additional elements to provide a more comprehensive perspective of SMA-driven CI. Further studies are needed to understand how customer sentiments are influenced by SM channels, how different SM channels influence the effectiveness of gathering CI, and how such differences affect the achievement of business value and decision-making efficiency from CI. 


\section{REFERENCES}

Balduini, M., Celino, I., Dell'Aglio, D., Della Valle, E., Huang, Y., Lee, T., Kim, S.-H., \& Tresp, V. (2012). BOTTARI: An augmented reality mobile application to deliver personalized and location-based recommendations by continuous analysis of social media streams. Journal of Web Semantics, 16, 33-41. doi:10.1016/j. websem.2012.06.004

Brandt, T., Bendler, J., \& Neumann, D. (2017). Social media analytics and value creation in urban smart tourism ecosystems. Information \& Management, 54(6), 703-713. doi:10.1016/j.im.2017.01.004

Bukhari, I., Wojtalewicz, C., Vorvoreanu, M., \& Dietz, J. E. (2012, November). Social media use for large event management: The application of social media analytic tools for the Super Bowl XLVI. In 2012 IEEE Conference on Technologies for Homeland Security (HST) (pp. 24-29). IEEE. doi:10.1109/THS.2012.6459821

Calof, J. (2017). Canadian competitive intelligence practices-A study of practicing strategic and competitive intelligence professionals Canadian members. Foresight, 19(6), 577-589. doi:10.1108/FS-07-2017-0024

Calof, J., Richards, G. S., \& Smith, J. E. (2016). Foresight, competitive intelligence and business analytics for developing and running better programmes. In L. Gokhberg, D. Meissner, \& A. Sokolov (Eds.), Deploying foresight for policy and strategy makers (pp. 161-180). Springer. doi:10.1007/978-3-319-25628-3_11

Chae, B. K. (2015). Insights from hashtag \#supply chain and Twitter Analytics: Considering Twitter and Twitter data for supply chain practice and research. International Journal of Production Economics, 165, 247-259. doi:10.1016/j.ijpe.2014.12.037

Chaffey, D. (2018). Global social media research summary 2018. Smart Insights. https://www.smartinsights. com/social-media-marketing/social-media-strategy/new-global-social-media-research/

Chang, Y.-C., Ku, C.-H., \& Chen, C.-H. (2019). Social media analytics: Extracting and visualizing Hilton hotel ratings and reviews from TripAdvisor. International Journal of Information Management, 48, 263-279. doi:10.1016/j.ijinfomgt.2017.11.001

Chen, J. S., \& Ching, R. K. (2004). An empirical study of the relationship of IT intensity and organizational absorptive capacity on CRM performance. Journal of Global Information Management, 12(1), 1-17. doi:10.4018/ jgim.2004010101

Cleland, D. I., \& King, W. R. (1975). Competitive business intelligence systems. Business Horizons, 18(6), 19-28. doi:10.1016/0007-6813(75)90036-1

Dishman, P. L., \& Calof, J. L. (2008). Competitive intelligence: A multiphasic precedent to marketing strategy. European Journal of Marketing, 42(7/8), 766-785. doi:10.1108/03090560810877141

Gruber, T., Szmigin, I., Reppel, A. E., \& Voss, R. (2008). Designing and conducting online interviews to investigate interesting consumer phenomena. Qualitative Market Research, 11(3), 256-274. doi:10.1108/13522750810879002

Guimaraes, T., Sato, O., \& Kitanaka, H. (1999). Comparing US \& Japanese companies on competitive intelligence, IS support, and business change. Journal of Global Information Management, 7(3), 41-49. doi:10.4018/ jgim.1999070104

Halawani, F. M., Soh, P. C., \& Halawani, Y. M. (2020). Social Media Utilisation and Business Performance of Hotels in Lebanon: Exploring the Moderating Effects of Hotel Classification. Journal of Global Information Management, 28(3), 58-76. doi:10.4018/JGIM.2020070104

He, W., Tian, X., Chen, Y., \& Chong, D. (2016). Actionable social media competitive analytics for understanding customer experiences. Journal of Computer Information Systems, 56(2), 145-155. doi:10.1080/08874417.20 16.1117377

He, W., Tian, X., Tao, R., Zhang, W., Yan, G., \& Akula, V. (2017). Application of social media analytics: A case of analyzing online hotel reviews. Online Information Review, 41(7), 921-935. doi:10.1108/OIR-07-2016-0201

He, W., Wu, H., Yan, G., Akula, V., \& Shen, J. (2015). A novel social media competitive analytics framework with sentiment benchmarks. Information \& Management, 52(7), 801-812. doi:10.1016/j.im.2015.04.006 
Iftikhar, R., \& Khan, M. S. (2020). Social Media Big Data Analytics for Demand Forecasting: Development and Case Implementation of an Innovative Framework. Journal of Global Information Management, 28(1), 103-120. doi:10.4018/JGIM.2020010106

Kim, Y., Dwivedi, R., Zhang, J., \& Jeong, S. R. (2016). Competitive intelligence in social media Twitter: iPhone 6 vs. Galaxy S5. Online Information Review, 40(1), 42-61. doi:10.1108/OIR-03-2015-0068

Köseoglu, M. A., Chan, E. S. W., Okumus, F., \& Altin, M. (2019). How do hotels operationalize their competitive intelligence efforts into their management processes? Proposing a holistic model. International Journal of Hospitality Management, 83, 283-292. doi:10.1016/j.jjhm.2018.11.007

Lee, I. (2018). Social media analytics for enterprises: Typology, methods, and processes. Business Horizons, 61(2), 199-210. doi:10.1016/j.bushor.2017.11.002

Lincoln, Y. S., \& Guba, E. G. (1985). Naturalistic inquiry. Sage (Atlanta, Ga.).

Marr, B. (2018). How much data do we create every day? the mind-blowing stats everyone should read. Forbes. https://www.forbes.com/sites/bernardmarr/2018/05/21/how-much-data-do-we-create-every-day-the-mindblowing-stats-everyone-should-read/\#beb110b60ba9

Morse, J. M. (2000). Editorial: Determining sample size. Qualitative Health Research, 10(3), 3-5. doi:10.1177/104973200129118183 PMID:10947476

Muninger, M.-I., Hammedi, W., \& Mahr, D. (2019). The value of social media for innovation: A capability perspective. Journal of Business Research, 95, 116-127. doi:10.1016/j.jbusres.2018.10.012

Patton, M. (1990). Qualitative evaluation and research methods. Sage (Atlanta, Ga.).

Pellissier, R., \& Nenzhelele, T. E. (2013). Towards a universal definition of competitive intelligence. South African Journal of Information Management, 15(2), 1-7. doi:10.4102/sajim.v15i2.559

Porter, M. E. (1980). Competitive strategy: Techniques for analyzing industries and competitors. Free Press.

Prescott, J. E. (1995). The evolution of competitive intelligence. International Review of Strategic Management, $6,71-90$.

Rathore, A. K., Ilavarasan, P. V., \& Dwivedi, Y. K. (2016). Social media content and product co-creation: An emerging paradigm. Journal of Enterprise Information Management, 29(1), 7-18. doi:10.1108/JEIM-06-20150047

Rialp-Criado, J., \& Rialp, A. (2020). Speed of Use of Social Media as an Antecedent of Speed of Business Internationalization. Journal of Global Information Management, 28(1), 142-166. doi:10.4018/JGIM.2020010108

Safarnia, H., Akbari, Z., \& Abbasi, A. (2011). Review of competitive intelligence \& competitive advantage in the industrial estates companies in the Kerman City: Appraisal and testing of model by Amos Graphics. International Business Management, 2(2), 47-61. doi:10.3968/j.ibm.1923842820110202.006

Schlagwein, D., \& Hu, M. (2017). How and why organisations use social media: Five use types and their relation to absorptive capacity. Journal of Information Technology, 32(2), 194-209. doi:10.1057/jit.2016.7

Sheng, J., Amankwah-Amoah, J., \& Wang, X. (2017). A multidisciplinary perspective of big data in management research. International Journal of Production Economics, 19, 97-112. doi:10.1016/j.ijpe.2017.06.006

Shollo, A., \& Galliers, R. D. (2016). Towards an understanding of the role of business intelligence systems in organisational knowing. Information Systems Journal, 26(4), 339-367. doi:10.1111/isj.12071

Singh, A., Shukla, N., \& Mishra, N. (2018). Social media data analytics to improve supply chain management in food industries. Transportation Research Part E, Logistics and Transportation Review, 114, 398-415. doi:10.1016/j.tre.2017.05.008

Štefániková, L., \& Masàrovà, G. (2014). The need of complex competitive intelligence. Procedia: Social and Behavioral Sciences, 110, 669-677. doi:10.1016/j.sbspro.2013.12.911

Strauss, A., \& Corbin, J. (1994). Grounded theory methodology: An overview. In N. K. Denzin \& Y. S. Lincoln (Eds.), Handbook of qualitative research (pp. 273-285). Sage. 
Xiang, Z., Du, Q., Ma, Y., \& Fan, W. (2017). A comparative analysis of major online review platforms: Implications for social media analytics in hospitality and tourism. Tourism Management, 58, 51-65. doi:10.1016/j. tourman.2016.10.001

Xu, X., Wang, X., Li, Y., \& Haghighi, M. (2017). Business intelligence in online customer textual reviews: Understanding consumer perceptions and influential factors. International Journal of Information Management, 37(6), 673-683. doi:10.1016/j.ijinfomgt.2017.06.004

Jiwat Ram has published his research work in top tier high impact factor journals including International Journal of Production Economics, Computers in Human Behaviour, and Enterprise Information Systems, among others. His published work has been well received and four of his published papers have ranked in Top25 most downloaded papers from ScienceDirect. His two papers have been ranked in Top 25 Most Cited articles as well. Jiwat has growing portfolio of publications on Social media and Big data, and recently he has published his research on Social media driven business innovations.

Changyu Zhang did her bachelor's from IBSS XJTLU and has published her work in conferences. One of her coauthored papers has been ranked in Top 25 most downloaded articles. 